\title{
THE APPLICATION OF THE THEORY OF ADMISSIBLE NUMBERS TO TIME SERIES WITH CONSTANT PROBABILITY*
}

\author{
BY \\ FRANCIS .REGAN

\section{INTRODUCTION}

The idea of admissible numbers in probability is a new one. Its development has come about during the past few years through the researches of Copeland. $\dagger$ Admissible numbers furnish a method for testing the consistency of the assumptions of the theory of probability and also serve as a guide for setting up sets of assumptions. The problem of testing consistency is a very extensive one, since in almost every branch of the theory of probability new assumptions are made.

This paper is an extension of the concept of admissibility to time series. A time series is a sequence of occurrences, which are represented by a set of points on a time axis. These points must satisfy a certain law; namely, that there is a definite probability of getting a point in any interval. This probability may vary according to the length of the interval, or according to the length of the interval and the position of the interval.

In order that a probability situation may have any meaning from the statistical point of view, it must be capable of being repeated a large number of times under similar circumstances. A type of time series which is dealt with here has these repetitions given directly by the time series. In order that this may be the case, the probability of obtaining a point in any interval must be a periodic function of the position of the interval; that is, the coordinate of the left hand extremity.

Since this set of points possesses the property necessary to use the statistical point of view of probability without any modifications, then if it is to satisfy the fundamental assumptions of the theory of probability, it is neces-

* Presented to the Society, April 15, 1933; received by the editors October 4, 1933. The author wishes to acknowledge his appreciation to Arthur H. Copeland, of the University of Michigan, for many helpful suggestions throughout the progress of the work.

$\dagger$ A. H. Copeland, Admissible numbers in the theory of probability, American Journal of Mathematics, vol. 50, No. 4, Oct., 1928. Independent event histories, loc. cit., vol. 51, No. 4, Oct., 1929. Admissible numbers in the theory of geometrical probability, loc. cit., vol. 53, No. 1, Jan., 1931. The theory of probability from the point of view of admissible numbers, Annals of Mathematical Statistics, vol. 3, No. 3, Aug., 1932. 
sary that sequences of successes and failures be represented by the digits of admissible numbers. The number of conditions imposed upon these points has the power of the continuum, since, for every interval, a different set of conditions is obtained. It is the purpose of this paper to show that these conditions are consistent and can be satisfied.

The time series will be represented by the set of points $\tau_{1}<\tau_{2}<\ldots$ $<\tau_{i}<\cdots$. Let $f(\alpha, \tau, t)$ be the probability of $\alpha$ points of the series lying in an interval of length $\tau$, beginning at time $t$. It follows from the past discussion, that it is necessary for $f(\alpha, \tau, t)$ to be periodic in $t$. The $k$ th interval $I_{k}$ of the series is defined as $t+(k-1) \Lambda<h \leqq t+\tau+(k-1) \Lambda$, where $\Lambda$ is a period or an integral multiple of a period and $t+\tau \leqq \Lambda$. Let $x(\alpha, \tau, t, \Lambda)$ be a number such that its $k$ th digit is one if there are exactly $\alpha$ points in $I_{k}$ and zero otherwise. Since this paper deals with constant probability, it may be seen that the probability of $\alpha$ points of the series lying in $\tau$ is independent of $t$, and that every $\Lambda$ is a period. In this case, the time series $\tau_{1}<\tau_{2}<\ldots$ $<\tau_{i}<\ldots$ will be constructed so that the number $x(\alpha, \tau, t, \Lambda)$ is an element of the set $A[f(\alpha, \tau, t)]^{*}$ for every $\alpha, \tau, t$ and $\Lambda$, where $\tau=m \cdot 2^{-\sigma+1}, t=r \cdot 2^{-\sigma+1}$ and $\Lambda=\rho \cdot 2^{-\sigma+1}$, where $\alpha, \rho, m, r$ and $\sigma$ are positive integers and $r+m \leqq \rho . \dagger$ The construction of this series that will be given is, in a sense, a numerical construction and is probably about the simplest mathematical construction possible.

The case where the probability of $\alpha$ points lying in $\tau$ is dependent on $t$, will be discussed in a subsequent paper.

\section{Probability of at least one point lying in an INTerval}

1. When the probability does not depend upon the beginning time, it is seen $\ddagger$ that the probability of $\alpha$ points lying in an interval of length $\tau$ is

$$
f(\alpha, \tau, 0)=\left[e^{-m \tau}(m \tau)^{\alpha}\right] / \alpha ! .
$$

Since $m$ is the ratio constant which determines the unit of time, there will be no loss in generality by choosing $m$ equal to one.

For the present, we shall be concerned with the case in which at least one point lies in $\tau$. A geometrical construction of this time series may be formed

* The set $A[f(\alpha, \tau, t)]$ is the set of all admissible numbers associated with the probability function $f(\alpha, \tau, t)$.

$\dagger$ It should be observed that the function $f(\alpha, \tau, t)$ is independent of $t$, hence $f(\alpha, \tau, t) \equiv f(\alpha, \tau, 0)$. Also the number $x(\alpha, \tau, t, \Lambda)$ is independent of $t$, since it is an element of the set $A[f(\alpha, \tau, t)]$; that is, the numbers $x(\alpha, \tau, 0, \Lambda)$ and $x(\alpha, \tau, t, \Lambda)$ are members of the same set $A[f(\alpha, \tau, 0)]$, provided that $\tau=m \cdot 2^{-\sigma+1}, \Lambda=\rho \cdot 2^{-\sigma+1}, t=0$ in the first number and $t=r \cdot 2^{-\sigma+1}$ in the second, where $r+m \leqq \rho$.

$\ddagger$ See Fry, Probability and its Engineering $U$ ses, pp. 216-27 and pp. 232-35. A more rigorous proof than given by Fry will be published later in a joint paper by the author and Professor A. H. Copeland. 
to illustrate the phenomena arising from the probability function $[1-f(0, \tau$, $0)$ ]. Construct a set of segments of length $\tau$ on a line. A set of points $\tau_{1}<\tau_{2}$ $<\cdots<\tau_{i}<\cdots$ is distributed along this line in such a manner that the probability of at least one point lying in an interval of length $\tau$ is $[1-f(0, \tau$, $0)$ ]. Here the $k$ th interval $I_{k}$ is $(k-1) \tau<h \leqq k \tau .^{*}$ In $I_{k}$, there may be no points or at least one. Let us define $\sim x(0, \tau, 0, \tau) \dagger$ such that its $k$ th digit is one if there is at least one point in $I_{k}$ and zero otherwise. The success ratio is

$$
p_{N}\left[\sim x_{\tau}\right]=\sum_{k=1}^{N} \frac{x^{(k)}}{N}
$$

and we demand that $p\left(\sim x_{\tau}\right)=1-f(0, \tau, 0)$, where

$$
p\left(\sim x_{\tau}\right)=\lim _{N \rightarrow \infty} p_{N}\left(\sim x_{\tau}\right) .
$$

A physical illustration of a time series of this character is the occurrences of quakes in a certain region. Let us take for example, the quakes actually occurring from 1490 A.D. to 1930 A.D. in the region which is now Mexico, assuming that records of such could have been kept. These data would be applicable to the above. Let $\tau$ represent the time span of ten years and the set of points properly graphed the quakes. Here $\sim x_{\tau}$ will be defined such that its $k$ th digit is one if there is at least one quake in the $k$ th decade and zero otherwise. The success ratio is

$$
p_{44}\left[\sim x_{\tau}\right]=\sum_{k=1}^{44} \frac{x^{(k)}}{44} .
$$

If these records of quakes were kept up indefinitely this success ratio would approach $\left(1-e^{-10 m}\right)$.

2. We have constructed a time series which illustrates exactly how a physical event would be dealt with. It becomes our problem to build up an imaginary series which logically follows from the physical, but one that satisfies the laws of admissibility. In testing the consistency of the assumptions made in developing the probability function, the time series must possess certain properties, one of which is an unlimited number of occurrences. These properties will be exhibited in the next paragraph where the series will be developed.

The time series may be represented by a set of points $\tau_{1}<\tau_{2}<\cdots<\tau_{i}$

* Here $t=0$ and $\tau=\Lambda$.

$\dagger$ The symbol $\sim$ means "not." Let $\backsim x(0, \tau, 0, \tau)$ be represented by $\sim x_{\tau}$.

$\ddagger$ See Copeland, Admissible numbers in the theory of probability, American Journal of Mathematics, vol. 50, p. 536 . 
$<\ldots$ on the positive $\tau$-axis. These points satisfy the following law. Let this axis be divided into periods or intervals and let the $k$ th interval $I_{k}$ be defined as $(k-1) \rho \cdot 2^{-\sigma+1}<h \leqq k \cdot \rho \cdot 2^{-\sigma+1}$, where $\rho$ and $\sigma$ are positive integers. We are considering the case where $t=0, \Lambda=\tau=\rho \cdot 2^{-\sigma+1}$. Let $\sim x_{\tau}$ be such that the $k$ th digit is one if there is at least one point of the time series in $I_{k}$ and zero otherwise. The points of the time series must possess the property that $p\left[\sim x_{\tau}\right]=\left[1-e^{-\tau}\right]$ for every $\rho$ and $\sigma$.

3. We shall now construct the imaginary series. We shall construct a finite set of points of the series in such a manner that the conditions described above are satisfied to a certain degree of approximation when applied to unit intervals. We will call this set of points the first stage. The set of points for the second stage will be selected in such a manner that the conditions will hold to a certain degree of approximation when applied to unit intervals or intervals of length one half. For the third stage, the conditions can be applied to intervals of length $1, \frac{1}{2}$, or $\frac{1}{4}$. We let $N_{1}$ be the number of intervals employed in the first stage. In the second stage, there will be $N_{2}$ unit intervals or $2 \cdot N_{2}$ half unit intervals, etc. The choice of numbers $N_{1}, N_{2}, \cdots, N_{s}, \cdots$ will be determined at a later point in the paper. We shall construct the $N_{1}$ contiguous unit intervals of the first stage on the $\tau$-axis, beginning at time zero, the origin. The points of the time series will occupy the mid-points of these intervals. Let $X_{1}$ be a member of the set $A\left(1-e^{-1}\right)$ and let the $i$ th unit interval contain a point of the time series if and only if the $i$ th digit of $X_{1}$ equals one. If $j_{i}$ is the smallest number $j$ such that $j \cdot p_{j}\left(X_{1}\right)=i$, the points which we have constructed have the coordinates $\tau_{i}=j_{i}-\frac{1}{2}$. We have now constructed the first $N_{1} \cdot p_{N_{1}}\left(X_{1}\right)$ points of the time series.

To the $N_{1}$ unit intervals we will add $2 \cdot N_{2}$ intervals of length $\frac{1}{2}$. We will allow the next set of points of the time series to occupy the mid-points of these intervals. Those intervals which will contain points of the time series will be determined by the digits of a number $X_{2}$ which is a member of the set $A\left(1-e^{-2^{-1}}\right)$. In general the $s$ th set of points will occupy the mid-points of $2^{s-1} \cdot N_{s}$ intervals of length $2^{-s+1}$. Those intervals which will contain points of the series will be determined by the digits of a number $X_{s}$ which is a member of the set $A\left(1-e^{-2^{-a+1}}\right)$.

Let $\nu_{s}=N_{1}+N_{2}+\cdots+N_{s-1}\left(\nu_{1}=0\right)$ and

$$
\gamma_{s}=\sum_{k=1}^{s-1} 2^{k-1} \cdot N_{k} \cdot p_{2}^{k-1} \cdot N_{k}\left(X_{k}\right)
$$

Then after the $s$ th set of points has been chosen, we have determined $\gamma_{s+1}$ points and these points all lie in the interval from 0 to $\nu_{s+1}$. Let $s_{i}$ be such that $\gamma_{s_{i}}<i \leqq \gamma_{s_{i}+1}$ and let $j_{i}$ be the smallest $j$ such that $j \cdot p_{j}\left(X_{s_{i}}\right)=i-\gamma_{s_{i}}$. Then the coordinates of the points of the time series are given by the equations 


$$
\tau_{i}=\nu_{s_{i}}+j_{i} \cdot 2^{-s_{i}+1}-2^{-s_{i}}
$$

For example suppose that $i=\gamma_{2}+1$; then $s_{i}=2$ and the point $\tau_{i}$ lies to the right of the point $\nu_{2}$. Let us suppose further that 3 is the smallest integer $j$ such that $j \cdot p_{j}\left(X_{2}\right)=i-\gamma_{s_{i}}=1$. Then $\tau_{i}$ is the mid-point of the third half unit interval to the right of the point $\nu_{2}$; that is, $\tau_{i}=\nu_{2}+3 \cdot 2^{-1}-2^{-2}$.

Let us consider a further example. The time series consists of the sequence of points $\tau_{1}, \tau_{2}, \cdots, \tau_{i}, \cdots$ and the associated number $\sim x(0,1,0,1)=\sim x_{1}$ has the following sequence of digits:

$$
\begin{aligned}
X_{1}^{(1)} X_{1}^{(2)} \cdots X_{1}^{\left(N_{1}\right)}\left(X_{2}^{(1)}\right. & \left.\vee X_{2}^{(2)}\right)\left(X_{2}^{(3)} \vee X_{2}^{(4)}\right) \cdots \\
& \left(X_{2}^{\left(2 N_{2}-1\right)} \vee X_{2}^{\left(2 N_{2}\right)}\right)\left(X_{3}^{(1)} \vee X_{3}^{(2)} \vee X_{3}^{(3)} \vee X_{3}^{(4)}\right) \cdots,
\end{aligned}
$$

where $X_{2}^{(1)} \vee X_{2}^{(2)}=1$ whenever one or both of the numbers is equal to one, and $X_{2}^{(1)} \vee X_{2}^{(2)}=0$ otherwise, etc. We have the equation $p\left(X_{1}\right)=1-e^{-1}$ and we will prove that

$$
\begin{aligned}
p\left[(1 / 2) X_{2} \vee\right. & \left.(2 / 2) X_{2}\right] \\
& =p\left[(1 / 4) X_{3} \vee(2 / 4) X_{3} \vee(3 / 4) X_{3} \vee(4 / 4) X_{3}\right]=\cdots=1-e^{-1} .
\end{aligned}
$$

We will show that, for a proper choice of the numbers $N_{1}, N_{2}, \cdots, N_{s}, \cdots$, $p\left(\sim x_{1}\right)=1-e^{-1}$. The numbers $\sim x_{1 / 2}, \sim x_{1 / 4}$, etc. can be treated in a similar manner.

4. The time series is defined when the numbers $N_{8}$ are defined. We shall prove the following theorem.

THEOREM 1. If the time series consists of the points $\tau_{1}, \tau_{2}, \tau_{3}, \cdots$ satisfying the conditions

$$
\tau_{i}=\nu_{\varepsilon:}+j_{i} \cdot 2^{-s_{i}+1}-2^{-s_{i}}, \quad \nu_{s}=N_{1}+N_{2}+\cdots+N_{s-1} \quad\left(\nu_{1}=0\right),
$$

$\left(H_{1}\right) s_{i}$ is such that $\gamma_{s_{i}}<i \leqq \gamma_{s i+1}$,

$$
\gamma_{s}=\sum_{k=1}^{s-1} 2^{k-1} \cdot N_{k} \cdot p_{2^{k-1} \cdot N_{k}}\left(X_{k}\right) \quad\left(\gamma_{1}=0\right),
$$

$X_{8}$ is a member of the set $A\left(1-e^{-2^{-a+1}}\right)$,

$j_{i}$ is the smallest $j$ such that $j \cdot p_{j}\left(X_{s_{i}}\right)=i-\gamma_{s_{i}}$,

then the numbers $N_{1}, N_{2}, \cdots, N_{s}, \cdots$ can be so chosen that for every $\tau$ satisfying the conditions

$\tau=\rho \cdot 2^{-\sigma+1}$,
$\left(H_{2}\right) \rho$ and $\sigma$ are positive integers and $0<\rho \leqq 2^{2 \sigma-1}$, the corresponding number $\sim x_{\tau}$ is an element of the set $A\left(1-e^{-r}\right)$. 
In order to prove this theorem, it is necessary to divide the time axis into periods of length $\tau$. Each period is composed of $\rho$ intervals, whose lengths are the same as those at the $\sigma$-stage, and the $k$ th period $I_{k}$ is $(k-1) \tau<h \leqq k \tau$. At this stage the number representing the event which succeeds if there is at least one point in $I_{k}$, is

$$
\left\{\sum_{q=1}^{\rho}\left(\frac{q}{\rho}\right) X_{\sigma} \vee\right\}, *
$$

which is an element of $A\left(1-e^{-r}\right)$. At the $(\sigma+1)$-stage, in order to represent the same $I_{k}$, it is necessary to increase the number of intervals by $\rho$, and hence double the number of operators. At this stage the required number is

$$
\left\{\sum_{q=1}^{2 \rho}\left(\frac{q}{2 \rho}\right) X_{\sigma+1} \vee\right\}
$$

which is also a member of the set $A\left(1-e^{-\tau}\right)$. Continuing this process to the $s$-stage, we have the number

$$
\left\{\sum_{q=1}^{2^{s-\sigma} \cdot \rho}\left(\frac{q}{2^{s-\sigma} \cdot \rho}\right) X_{s} v\right\}
$$

which is an element of the set $A\left(1-e^{-\tau}\right)$. Let this number be represented by $U_{s}$. When $s \geqq \sigma$ and $\nu_{s} / \tau<k \leqq \nu_{s+1} / \tau$, then there exists at least one point in $I_{k}$ if and only if the digit $k-\nu_{s} / \tau$ of $U_{s}$ is one. Hence, when $s \geqq \sigma$ the digits $\left(\nu_{s} / \tau\right)+1$ to $\nu_{s+1} / \tau$ of $\sim x_{\tau}$ are the same as the digits 1 to $N_{s} / \tau$ of $U_{s}$. We shall now show that this is actually the case when $s \geqq \sigma$.

The numbers $N_{s}$ heretofore mentioned are chosen so that $\nu_{s} /(n \tau)$ is an integer, if $n \leqq s, \sigma \leqq s$, and $0<\rho \leqq 2^{2 \sigma-1}$, from which follows that $N_{s} /(n \tau)$ is an integer. If the $k$ th digit of $X_{s}$ is one, then the $k$ th interval at the $s$-stage contains a point of the time series. There are $2^{s-1} \cdot N_{s}$ intervals, and hence we use $2^{\varepsilon-1} \cdot N_{s}$ digits of $X_{s}$. We may express $X_{s}$ as

$$
X_{s}=. X_{s}^{(1)} X_{s}^{(2)} \cdots X_{s}^{\left(2^{s-\sigma} \cdot N_{s}\right)} \cdots,
$$

from which we may form

$$
\left(\frac{1}{\rho \cdot 2^{s-\sigma}}\right) X_{s}=. X_{s}^{(1)} X_{s}^{\left(\rho \cdot 2^{s-\sigma}+1\right)} \cdots X_{s}^{\left.\left(N_{s} / \tau-1\right)_{\rho \cdot 2^{s} \cdot \sigma}+1\right)} \cdots,
$$

$$
\left(\frac{\rho \cdot 2^{s-\sigma}}{\rho \cdot 2^{s-\sigma}}\right) X_{s}=. X_{s}^{\left(\rho \cdot 2^{s-\sigma}\right)} X_{s}^{\left(2 \rho \cdot 2^{s-\sigma}\right)} \cdots X_{s}^{\rho^{\rho^{-1}} N_{s}} \cdots .
$$

* This symbol is used to represent the number $\left\{(1 / \rho) X_{\sigma} \vee(2 / \rho) X_{\sigma} \vee \cdots \vee(\rho / \rho) X_{\sigma}\right\}$. In general, let $\left\{\sum_{i=1}^{n}(i / n) Y \vee\right\}$ represent the number $\{(1 / n) Y \vee \cdots \vee(n / n) Y\}$.

† See Lemma 1 in the second paragraph below. 
We are considering $N_{\mathrm{s}} / \tau$ digits of each of these numbers. It may be seen from these numbers that the first digit of $U_{\mathrm{s}}$ is one if and only if at least one of the digits $X_{s}^{(1)}, X_{s}^{(2)}, \cdots, X_{s}^{\left(\rho \cdot 2^{5-\sigma}\right)}$ is one. By analyzing the digits $X_{s}^{(1)}$ to $X_{s}^{\left(\rho \cdot 2^{\rho-\sigma}\right)}$ inclusive, it is seen that if at least one of these is one, then there is at least one point of the time series in the first period of the $s$-stage; that is, of the interval $I_{\left(\nu_{\mathrm{s}} / \tau\right)+1}$. Hence the $\left\{\left(\nu_{\mathrm{s}} / \tau\right)+1\right\}$ th digit of $\sim x_{\tau}$ is the same as the first digit of $U_{s}$. This process may be continued for the $N_{s} / \tau$ digits of $U_{\text {s. }}$

Previously, we made use of the following lemma:

LEMMA 1. If $X_{8}$ is an element of $A(p)$, the number $\left\{\sum_{q=1}^{n}(q / n) X_{8} \vee\right\}$ is a member of the set $A\left[1-(1-p)^{n}\right]$.

We know that

$$
\sim \sum_{q=1}^{n}\left(\frac{q}{n}\right) X_{s} \vee=\prod_{q=1}^{n} \sim\left(\frac{q}{n}\right) X_{*} \cdot *^{*}
$$

Since the numbers $(q / n) X_{s}(q=1,2, \cdots, n)$ are independent, then $\sim(q / n) X_{s}$ $(q=1,2, \cdots, n)$ are independent. $\dagger$ From (a), we get

$$
\sum_{q=1}^{n}\left(\frac{q}{n}\right) X_{\bullet} \vee=\sim \prod_{q=1}^{n} \sim\left(\frac{q}{n}\right) X_{\bullet} \cdot
$$

and hence, we have

$$
p\left[\sum_{q=1}^{n}\left(\frac{q}{n}\right) X_{s} v\right]=1-p\left[\prod_{q=1}^{n} \sim\left(\frac{q}{n}\right) X_{0} \cdot\right],
$$

or

$$
p\left[\sum_{q=1}^{n}\left(\frac{q}{n}\right) X_{8} v\right]=\left[1-(1-p)^{n}\right]
$$

We see that $\sum_{q=1}^{n}(q / n) X_{\varepsilon} \vee$ has the desired probability, but we must now show that

$$
p\left[\prod_{i=1}^{k}\left(\frac{r_{i}}{m}\right)\left\{\sum_{q=1}^{n}\left(\frac{q}{n}\right) X_{\mathbf{a}} v\right\} \cdot\right]=\left[1-(1-p)^{n}\right]^{k},
$$

* Here the symbol $\Pi \backsim(q / n) X_{s} \cdot$ represents the number $\left\{\backsim(1 / n) X_{8} \cdot \backsim(2 / n) X_{0} \cdots \cdots \backsim(n / n) X_{s}\right\}$. Such symbols used in this paper will have similar meanings. For the truth of this equality, see Copeand, The theory of probability from the point of view of admissible numbers, Annals of Mathematical Statistics, vol. 3, Aug., 1932, p. 149.

† See Copeland, Admissible numbers in the theory of probability, American Journal of Mathematics, Oct., 1928, Theorem 5, p. 542. 
for every set of numbers, $r_{1}, r_{2}, \cdots, r_{k}$, such that $0<r_{i} \leqq m$ and $r_{i} \neq r_{j}$ if $i \neq j$. Since

$$
\sum_{q=1}^{n}\left(\frac{q}{n}\right) X_{\mathrm{s}} \vee=\sim \prod_{q=1}^{n} \sim\left(\frac{q}{n}\right) X_{\mathrm{s}} \cdot
$$

then

$$
\left(\frac{r_{i}}{m}\right)\left[\sum_{q=1}^{n}\left(\frac{q}{n}\right) X_{0} v\right]=\sim \prod_{q=1}^{n} \sim\left(\frac{q+\left(r_{i}-1\right) n}{m n}\right) X_{\bullet} \cdot \cdot^{*}
$$

Then

$$
\prod_{i=1}^{k}\left(\frac{r_{i}}{m}\right)\left[\sum_{q=1}^{n}\left(\frac{q}{n}\right) X_{i} \vee\right]=\prod_{i=1}^{k} \sim \prod_{q=1}^{n} \sim\left(\frac{q+\left(r_{i}-1\right) n}{m n}\right) X_{s} \cdot .
$$

The numbers $r_{i}$ are chosen such that for every set $r_{1}, r_{2}, \cdots, r_{k}$, we have $0<r_{i} \leqq m$ and $r_{i} \neq r_{i}$ if $i \neq j$. Then the numbers $\sim\left\{\left[q+\left(r_{i}-1\right) n\right] /(m n)\right\} X_{8}$ are independent, and hence the numbers $\Pi_{q=1}^{n} \sim\left\{\left[q+\left(r_{i}-1\right) n\right] /(m n)\right\} X_{\bullet} \cdot \dagger$ are independent. We now conclude that

$$
p\left[\prod_{i=1}^{k}\left(\frac{r_{i}}{m}\right)\left\{\sum_{q=1}^{n}\left(\frac{q}{n}\right) X_{s} \vee\right\} \cdot\right]=\left[1-(1-p)^{n}\right]^{k} .
$$

Therefore the lemma is proved.

The following fundamental lemma will enable us to determine the choice of the numbers $N_{8}$.

LEMMA 2. If there exists a sequence $U_{1}, U_{2}, \cdots, U_{s}, \cdots$ and a monotonic non-increasing sequence $\epsilon_{s}$ such that $\lim _{s \rightarrow \infty} \epsilon_{s}=0$, and if $H_{1}, H_{2}, \cdots, H_{s}, \cdots$ and $J_{1}, J_{2}, \cdots, J_{s}, \cdots$ are two sets of integers such that

$$
\left|p_{N}\left(U_{s+1}\right)-p\right|<\epsilon_{s} / 3 \text {, if } N \geqq H_{s}
$$

and

$$
\left|p_{N}\left(U_{s}\right)-p\right|+\left[\mu_{s}+H_{s}\right] / J_{s}<\epsilon_{s} / 3 \text {, if } N \geqq J_{s},
$$

where $\mu_{s}=J_{1}+J_{2}+\cdots+J_{s-1}\left(\mu_{1}=0\right)$, and if $x$ is such that its digits $\mu_{s}+1$ to $\mu_{s+1}$ are the same as the digits 1 to $J_{s}$ of $U_{s}$, where $s_{0} \leqq s$, then

$$
\left|p_{N}(x)-p\right|<\epsilon_{s},
$$

provided $\mu_{s+1} \leqq N \leqq \mu_{s+2}$ and $s_{0} \leqq s$.

* See Copeland, Admissible numbers in the theory of probability, American Journal of Mathematics, Oct., 1928, p. 539.

† Since these numbers are independent, their negations are also. 
By hypothesis, the digits $\mu_{s}+1$ to $\mu_{s+1}$ of $x$ are the same as the digits 1 to $J_{\text {s of }} U_{s}$, where $s_{0} \leqq s$. Hence, we have

$$
x=x^{(1)} x^{(2)} \cdots x^{(k)} \cdots x^{\left(\mu_{0}\right)} \cdots x^{\left(\mu_{t}+1\right)} \cdots x^{\left(\mu_{t}+1\right)} \ldots
$$

and

$$
U_{s}=\cdot u_{s}^{(1)} u_{s}^{(2)} \cdots u_{s}^{(k)} \cdots u_{s}^{\left(s_{s}\right)} \cdots \text {, }
$$

where the $x^{(k)}$ and $u_{s}^{(k)}$ are ones or zeros and $x^{\left(\mu_{s}+1\right)}=u_{s}^{(1)}, x^{\left(\mu_{0}+2\right)}=u_{s}^{(2)}$, $\cdots, x^{\left(\mu_{s+1}\right)}=u_{s}^{J}$, where $s_{0} \leqq s .{ }^{*}$

Since

$$
p_{N}(x)=\sum_{k=1}^{N} \frac{x^{(k)}}{N} \text { and } p_{J_{\theta}}\left(U_{\theta}\right)=\sum_{k=1}^{J_{b}} \frac{u_{\theta}^{(k)}}{J_{s}},
$$

it follows that

$$
\left|N \cdot p_{N}(x)-J_{s} \cdot p_{J_{s}}\left(U_{8}\right)\right| \leqq N-J_{s},
$$

when $N \geqq \mu_{8+1}$.

Hence

(e)

$$
\left|p_{N}(x)-\left[\frac{J_{s}}{N}\right] p_{J_{0}}\left(U_{s}\right)\right| \leqq \frac{N-J_{s}}{N} \leqq \frac{\mu_{s}+H_{s}}{J_{s}}<\frac{\epsilon_{s}}{3},
$$

if $\mu_{s+1} \leqq N \leqq \mu_{s+1}+H_{s}$.

Combining (b) and (e), we obtain

$$
\left|p_{N}(x)-\left[\frac{J_{s}}{N}\right] p\right|<\frac{\epsilon_{s}}{3}\left[1+\left(\frac{J_{s}}{N}\right)\right] \leqq 2 \frac{\epsilon_{s}}{3} .
$$

But

(g)

$$
\left|p-\left[\frac{J_{s}}{N}\right] p\right|=\frac{N-J_{s}}{N} \cdot p<\frac{\mu_{s}+H_{s}}{J_{s}}<\frac{\epsilon_{s}}{3},
$$

if $\mu_{s+1} \leqq N \leqq \mu_{s+1}+H_{s}$.

Therefore, adding (f) and (g) we get

(h)

$$
\left|p_{N}(x)-p\right|<\epsilon_{s}
$$

if $\mu_{s+1} \leqq N \leqq \mu_{s+1}+H_{s}$.

We also know that

$$
p_{N-\mu_{\theta+1}}\left(U_{\theta+1}\right)=\sum_{k=1}^{N-\mu_{s+1}} \frac{u_{\theta+1}^{(k)}}{N-\mu_{\theta+1}},
$$

provided $N \geqq \mu_{s+1}$.

* It may be noted that the first $\mu_{s_{0}}$ digits may be arbitrarily defined for $x$. 
Using (d) and (i), we may form

$$
\left|N p_{N}(x)-J_{s} p_{J_{t}}\left(U_{s}\right)-\left(N-\mu_{\theta+1}\right) p_{N-\mu_{s+1}}\left(U_{\theta+1}\right)\right|,
$$

and find for what values of $N$ this expression will be less than $N \epsilon_{s} / 3$. We find that

$$
\text { (k) } \begin{aligned}
\mid p_{N}(x)-\left[J_{s} / N\right] p_{J_{s}}\left(U_{s}\right)- & \left(\left[N-\mu_{s+1}\right] / N\right) p_{N-\mu_{s+1}}\left(U_{s+1}\right) \mid \\
& \leqq \\
& {\left[N-J_{s}-\left(N-\mu_{s+1}\right)\right] / N=\mu_{s} / N \leqq \epsilon_{s} / 3, }
\end{aligned}
$$

if $\mu_{s+1}+H_{s} \leqq N \leqq \mu_{s+2}$.

Combining (a), (b) and (k), we obtain

$$
\left|p_{N}(x)-\left[\left\{J_{s}+N-\mu_{s+1}\right\} / N\right] p\right|<\left[2-\mu_{s} / N\right] \epsilon_{s} / 3<2 \epsilon_{s} / 3 .
$$

But

(m)

$$
\left|p-\left[\left\{J_{s}+N-\mu_{\theta+1}\right\} / N\right] p\right|=\left(\mu_{s} / N\right) p<\epsilon_{s} / 3,
$$

if $\mu_{s+1}+H_{s} \leqq N \leqq \mu_{s+2}$.

Adding (l) and (m), we get

(n)

$$
\left|p_{N}(x)-p\right|<\epsilon_{s}
$$

if $\mu_{s+1}+H_{s} \leqq N \leqq \mu_{s+2}$.

It follows from (h) and (n) that

$$
\left|p_{N}(x)-p\right|<\epsilon_{\theta},
$$

provided $\mu_{s+1} \leqq N \leqq \mu_{s+2}$, where $s_{0} \leqq s$.

In order to prove Theorem 1, we shall put it in the form of the fundamental lemma.

Since $U_{s}$ is an element of $A\left(1-e^{-r}\right)$, it follows that

$$
p\left[\prod_{i=1}^{k}\left(\frac{r_{i}}{n}\right) U_{\cdot} \cdot\right]=\left[1-e^{-r}\right]^{k} .
$$

Let $\epsilon_{1}, \epsilon_{2}, \cdots, \epsilon_{s}, \cdots$ be a decreasing sequence of positive numbers having the limit zero. We can choose two sets of integers $M_{1}, M_{2}, \cdots, M_{s}, \cdots$ and $N_{1}, N_{2}, \cdots, N_{0}, \cdots$ such that

$$
\left|p_{N}\left[\prod_{i=1}^{k}\left(\frac{r_{i}}{n}\right) U_{\theta+1} \cdot\right]-\left[1-e^{-\tau}\right]^{k}\right|<\frac{\epsilon_{s}}{3},
$$

whenever $N \geqq M_{8} /(n \tau)$, and

$$
\left|p_{N}\left[\prod_{i=1}^{k}\left(\frac{r_{i}}{n}\right) U_{s} \cdot\right]-\left[1-e^{-\tau}\right] k\right|+\frac{\nu_{s}+M_{s}}{N_{s}}<\frac{\epsilon_{s}}{3},
$$


when $N \geqq N_{s} /(n \tau)$, and where $\nu_{s}=N_{1}+N_{2}+\cdots+N_{s-1}$. The sequence $M_{s}$ must be chosen so that $M_{s} /(n \tau)$ is an integer. It has been stated that $\nu_{s} /(n \tau)$ and $N_{s} /(n \tau)$ are integers.

It is understood that conditions $\left(1^{\prime}\right)$ and $\left(2^{\prime}\right)$ hold for every set of positive integers $n, \sigma, \rho, r_{1}, r_{2}, \cdots, r_{k}$, such that $n \leqq s, \sigma \leqq s, 0<\rho \leqq 2^{2 \sigma-1}, r_{i} \leqq n$ and $k \leqq n$, where $r_{i} \neq r_{j}$ if $i \neq j$.

The first $\nu_{\sigma} / \tau$ digits of $\sim x_{\tau}$ are found directly from the time series, for instance the first digit of $\sim x_{\tau}$ is one if there is at least one point of the time series in $I_{1}$. When $\sigma \leqq s$, we have shown that the digits $\left(\nu_{s} / \tau\right)+1$ to $\nu_{s+1} / \tau$ of $\sim x_{\tau}$ are the same as the digits 1 to $N_{s} / \tau$ of $U_{s}$. Then the digits $\left(\nu_{s} /(n \tau)\right)+1$ to $\nu_{s+1} /(n \tau)$ of $\left[\Pi_{i=1}^{k}\left(r_{i} / n\right) \sim x_{\tau} \cdot\right]$ are the same digits as 1 to $N_{s} /(n \tau)$ of $\left[\Pi_{i=1}^{k}\left(r_{i} / n\right) U_{s} \cdot\right]$.

We may now make the comparison of the theorem with the lemma as follows. The numbers

and

$$
\left[\prod_{i=1}^{k}\left(\frac{r_{i}}{n}\right) U_{s} \cdot\right],\left[1-e^{-\tau}\right]^{k}, \frac{M_{s}}{n \tau}, \frac{N_{s}}{n \tau}, \frac{\nu_{s}}{n \tau}, \sigma
$$

$$
\left[\prod_{i=1}^{k}\left(\frac{r_{i}}{n}\right) \sim x_{\tau} \cdot\right]
$$

have taken the places of $U_{s}, p, H_{s}, J_{s}, \mu_{s}, s_{0}$ and $x$ respectively. The numbers $N_{s}$ have now been selected and hence the time series is determined so that for every $\rho$ and $\sigma, \sim x_{\tau}$ is an element of $A\left(1-e^{-r}\right)$.

5. Since a time series has been obtained so that the conditions of admissibility are satisfied in the period $\tau$, it is natural to inquire whether similar conditions hold when, within the period $\tau$, sub-intervals of the form $2^{-\sigma+1}$ are omitted from consideration.

We shall consider $m$ intervals of the form $2^{-\sigma+1}$ in the period $\tau$. Let these intervals begin at $\rho_{i} \cdot 2^{-\sigma+1}, i=1,2, \cdots, m$, where $\rho_{m}+1 \leqq \rho$ and $m \leqq \rho$. The sub-intervals of $I_{k}$ will be defined as follows: $(k-1) \tau+\rho_{i} \cdot 2^{-\sigma+1}<h \leqq(k-1) \tau$ $+\left(\rho_{i}+1\right) 2^{-\sigma+1}, i=1,2, \cdots, m$. Corresponding to $\sim x_{r}$ of $\S 2$, we defined a number $\sim x_{\tau^{\prime}}{ }^{*}$ such that its $k$ th digit is one if there exists at least one point of the time series in the $m$ intervals of $I_{k}$ and zero otherwise. The points of the time series must possess the property that $p\left[\sim x_{r^{\prime}}\right]$ is equal to $\left(1-e^{-r^{\prime}}\right)$, for every integral value of $m, \sigma$ and $\rho$, where $m \leqq \rho$.

Since the same type of time series is used for this case, then this series is defined when the corresponding numbers $N_{\mathrm{s}}$ are defined. $\dagger$

With these remarks, we come to

* This symbol written in full is $\sim x\left(0, \tau^{\prime}, t, \Lambda\right)$, where $\tau^{\prime}=m \cdot 2^{-\sigma+1}, t=0$ and $\Lambda=\tau=\rho \cdot 2^{-\sigma+1}$.

$\dagger$ The numbers $N$, used here are not necessarily the same numerically as in the preceding discussion. 
THEOREM 2. If the conditions $\left(H_{1}\right)$ of Theorem 1 are satisfied, then the numbers $N_{1}, N_{2}, \cdots, N_{8}$ can be so chosen that for every $\tau$ and $\tau^{\prime}$ satisfying the conditions

$$
\begin{aligned}
\tau & =\rho \cdot 2^{-\sigma+1}, \\
\tau^{\prime} & =m \cdot 2^{-\sigma+1},
\end{aligned}
$$

where $m, \rho$ and $\sigma$ are positive integers, $m \leqq \rho$ and $0<\rho \leqq 2^{2 \sigma-1}$, the corresponding number $\sim x_{\tau^{\prime}}$ is an element of the set $A\left(1-e^{-\tau^{\prime}}\right)$.

In proving this theorem, we employ the following scheme. The number which characterizes the $m$ intervals at the $\sigma$-stage for the event that succeeds if there is at least one point in the $m$ intervals of $I_{k}$, is

$$
\left\{\left[\left(\rho_{1}+1\right) / \rho\right] X_{\sigma} \vee\left[\left(\rho_{2}+1\right) / \rho\right] X_{\sigma} \vee \cdots \vee\left[\left(\rho_{m}+1\right) / \rho\right] X_{\sigma}\right\},
$$

which is an element of $A\left(1-e^{-\tau^{\prime}}\right)$, which follows from Lemma 1 . At the $s-$ stage, the number that characterizes this event for the $m$ intervals of $I_{k}$ is

$$
\begin{gathered}
{\left[\sum _ { i = 1 } ^ { m } \left\{\left[\left(\rho_{i} \cdot 2^{s-\sigma}+1\right) /\left(\rho \cdot 2^{s-\sigma}\right)\right] \vee\left[\left(\rho_{i} \cdot 2^{s-\sigma}+2\right) /\left(\rho \cdot 2^{s-\sigma}\right)\right] \vee \cdots\right.\right.} \\
\vee \\
\left.\left.\vee\left[\left(\rho_{i} \cdot 2^{s-\sigma}+2^{s-\sigma}\right) /\left(\rho \cdot 2^{s-\sigma}\right)\right] \vee\right\}\right] X, \vee,
\end{gathered}
$$

which is an element of $A\left(1-e^{-\tau^{\prime}}\right)$. Let this number be represented by $W_{s}$. When $s \geqq \sigma$ and $\nu_{s} / \tau<k \leqq \nu_{s+1} / \tau$, then there exists at least one point in the $m$ intervals of $I_{k}$ if and only if the $\left(k-\nu_{s} / \tau\right)$ th digit of $W_{s}$ is one. Hence, when $s \geqq \sigma$, the digits $\left(\nu_{s} / \tau\right)+1$ to $\nu_{s+1} / \tau$ of $\sim x_{\tau^{\prime}}$ are the same as the digits 1 to $N_{s} / \tau$ of $W_{s}$. The numbers $N_{s}$ are chosen so that $\nu_{s} /(n \tau)$ is an integer, if $n \leqq s$, $\sigma \leqq s$, and $0<\rho \leqq 2^{2 \sigma-1}$.

Let $\epsilon_{\mathrm{s}}$ be a decreasing sequence of positive numbers having the limit zero. We can select a set of integers $M_{1}, M_{2}, \cdots, M_{s}, \cdots$, such that

$$
\left|p_{N}\left\{\prod_{i=1}^{k}\left(\frac{r_{i}}{n}\right) W_{\theta+1} \cdot\right\}-\left\{1-e^{-\tau^{\prime}}\right\}^{k}\right|<\frac{\epsilon_{s}}{3},
$$

if $N \geqq M_{s} /(n \tau)$, where the sequence $M_{s}$ has been chosen so that $M_{s} /(n \tau)$ is an integer. We can choose a second set of integers $N_{1}, N_{2}, \cdots, N_{\ell}, \cdots$, such that

$$
\left|p_{N}\left\{\prod_{i=1}^{k}\left(\frac{r_{i}}{n}\right) W_{s} \cdot\right\}-\left\{1-e^{-\tau^{\prime}}\right\} k\right|+\frac{\nu_{s}+M_{s}}{N_{s}}<\frac{\epsilon_{s}}{3},
$$

when $N \geqq N_{s} /(n \tau)$ and $\nu_{s}=N_{1}+N_{2}+\cdots+N_{s-1}$. 
Conditions (a) and (b) hold for every $\rho_{1}, \rho_{2}, \cdots, \rho_{m}, m, n, \rho$ and $\sigma$ such that $\rho_{m}+1 \leqq \rho, m \leqq \rho, n \leqq s, 0<\rho \leqq 2^{2 \sigma-1}, \sigma \leqq s$, and for every set of numbers, $r_{1}, r_{2}, \cdots, r_{k}$, such that $r_{i} \leqq n, k \leqq n$, where $r_{i} \neq r_{j}$ if $i \neq j$.

When $\sigma \leqq s$, we see that the digits $\left(\nu_{s} / \tau\right)+1$ to $\nu_{s+1} / \tau$ of $\sim x_{\tau^{\prime}}$ are the same as the digits 1 to $N_{s} / \tau$ of $W_{s}$. It will be seen from the definition of $\sim x_{\tau^{\prime}}$, that the digits 1 to $\nu_{\sigma} / \tau$ of $\sim x_{\tau^{\prime}}$ have been determined from the $m$ intervals of $I_{1}$ to $I_{v_{\sigma} / \tau}$. The digits $\left(\nu_{s} /(n \tau)\right)+1$ to $\nu_{s+1} /(n \tau)$ of $\left[\Pi_{i=1}^{k}\left(r_{i} / n\right) \sim x_{\tau^{\prime}} \cdot\right]$ are the same as 1 to $N_{s} /(n \tau)$ of $\left[\Pi_{i=1}^{k}\left(r_{i} / n\right) W_{s} \cdot\right]$.

The fundamental lemma may now be applied. The numbers

$$
\begin{array}{r}
{\left[\prod_{i=1}^{k}\left(r_{i} / n\right) W_{s} \cdot\right],\left[1-e^{-r^{\prime}}\right]^{k}, M_{s} /(n \tau), N_{s} /(n \tau), \nu_{s} /(n \tau), \sigma} \\
\text { and }\left[\prod_{i=1}^{k}\left(r_{i} / n\right) \sim x_{\tau^{\prime}} \cdot\right]
\end{array}
$$

have taken the places of $U_{s}, p, H_{s}, J_{s}, \mu_{s}, s_{0}$ and $x$ respectively. This shows that the numbers $N_{s}$ have been chosen so that the time series is definitely determined in such a way that for every $m, \rho$, and $\sigma$, where $m \leqq \rho, \sim x_{r^{\prime}}$ is an element of $A\left(1-e^{-\tau^{\prime}}\right)$.

If $m=\rho$, then $\rho_{1}=0$ and this case reduces to Theorem 1. Furthermore, if $\rho_{1}=r$ and $\rho_{m}=r+m-1$, then $\sim x_{\tau^{\prime}}$ becomes $\sim x(0, \tau, t, \Lambda)$ where $\tau=m \cdot 2^{-\sigma+1}$, $t=r \cdot 2^{-\sigma+1}$ and $\Lambda=\rho \cdot 2^{-\sigma+1}$.

\section{Probability of $\alpha$ PoINTS LYING IN AN INTERVAL}

6. The same type of time series that was defined in $\$ 2$ will be used here. As formerly, the intervals are closed on the right and open on the left. The $k$ th interval $I_{k}$ is $(k-1) \tau<h \leqq k \tau$. Let us now define the number $x(\alpha, \tau, 0, \tau)^{*}$ such that its $k$ th digit is one if exactly $\alpha$ points lie in $I_{k}$ and zero otherwise. The points of the time series must be distributed on the time axis so that $p\left[x_{\tau}\right]$ is equal to $\left[\left(\tau^{\alpha} e^{-r}\right) / \alpha !\right]$, for every integral value of $\rho, \sigma$ and $\alpha$.

7 . As before, the numbers $N_{8}$ must be chosen so that a consistent time series will be defined. The fundamental theorem here is the following:

THEOREM 3. If the conditions $\left(H_{1}\right)$ of Theorem 1 are satisfied, then the numbers $N_{1}, N_{2}, \cdots, N_{s}, \cdots$ can be chosen for every $\tau$ satisfying the conditions

$$
\tau=\rho \cdot 2^{-\sigma+1}
$$

$\rho, \alpha$ and $\sigma$ are positive integers, where $0<\rho \leqq 2^{2 \sigma-1}$, so that the corresponding number $x_{\tau}$ is a member of the set $A\left[\left(\tau^{\alpha} e^{-r}\right) / \alpha !\right]$.

Let us define $\rho, \tau, \beta_{s}$ and $\alpha$, such that $0<\rho \leqq 2^{2 \sigma-1}, \tau=\rho \cdot 2^{-\sigma+1}, \beta_{s}=\rho \cdot 2^{s-\sigma}$

* Let $x(\alpha, \tau, 0, \tau)$ be denoted by $x_{\tau}$. 
and $\alpha \leqq \beta_{s}$. At the $s$-stage there are $\beta_{s}$ divisions of $\tau$. Let us consider an event which succeeds if there is a point in each of $\alpha$ such intervals and no points in the remaining $\left(\beta_{s}-\alpha\right)$ intervals. The number corresponding to this situation is

$$
U_{s}=\sum^{\beta_{s} C_{\alpha}}\left\{\prod_{i=1}^{\alpha}\left(\frac{q_{i}}{\beta_{s}}\right) X_{s} \cdot \prod_{i=\alpha+1}^{\beta_{8}} \sim\left(\frac{q_{i}}{\beta_{s}}\right) X_{s} \cdot\right\} \vee, *
$$

where $q_{i} \neq q_{i^{\prime}}$ if $i \neq i^{\prime}$ in every term of the symbolic sum ( $\left.v\right)$. This number $U_{s}$ is admissible, $\dagger$ and is a member of the set $A\left[{ }_{\beta_{c}} C_{\alpha}\left(1-e^{-\tau / \beta_{o}}\right)^{\alpha}\left(e^{-\tau / \beta_{s}}\right)^{\beta_{\theta}-\alpha}\right]$. Since

$$
\lim _{s \rightarrow \infty} \beta_{s} C_{\alpha}\left(1-e^{-\tau / \beta_{s}}\right)^{\alpha}\left(e^{\left.-\tau / \beta_{s}\right)^{\beta_{s}-\alpha}}=\left[\tau^{\alpha} e^{-\tau}\right] / \alpha !, \ddagger\right.
$$

we can choose $s$ and $N$ in such a manner that the difference

$$
p_{N}\left\{\prod_{i=1}^{k}\left(\frac{r_{i}}{n}\right) U_{\cdot} \cdot\right\}-\left\{\left[\tau^{\alpha} e^{-r}\right] / \alpha !\right\}^{k}
$$

is arbitrarily small. We have to satisfy conditions analogous to $\left(1^{\prime}\right)$ and $\left(2^{\prime}\right)$ of $\$ 4$ but since

$$
{ }_{\beta_{s}} C_{\alpha}\left(1-e^{-\tau / \beta_{s}}\right)^{\alpha}\left(e^{-\tau / \beta_{s}}\right)^{\beta_{s}-\alpha} \neq\left(\tau^{\alpha} e^{-\tau}\right) / \alpha !
$$

we have an additional problem. The sequence $\epsilon_{s}$ can no longer be arbitrary and the conditions analogous to $\left(1^{\prime}\right)$ and $\left(2^{\prime}\right)$ cannot hold for every $s$ which is greater than or equal to $\sigma$. We will show that there exists a monotonic nonincreasing sequence $\epsilon_{s}$ such that $\lim _{s \rightarrow \infty} \epsilon_{s}=0$ and two sequences of integers $M_{1}, M_{2}, \cdots, M_{s}, \cdots$ and $N_{1}, N_{2}, \cdots, N_{s}, \cdots$ and a function $f(s)$ with an inverse $f^{-1}(\sigma)$ such that if $\sigma \leqq f(s)$ or $\sigma \leqq f^{-1}(\sigma) \leqq s$, then the condition analogous to $\left(1^{\prime}\right)$ and $\left(2^{\prime}\right)$ holds. $\S$ When $s \geqq f^{-1}(\sigma) \geqq \sigma$ and $\nu_{s} / \tau<k \leqq \nu_{s+1} / \tau$, then there will be exactly $\alpha$ points in $I_{k}$ if and only if the digit $\left(k-\nu_{s} / \tau\right)$ of $U$, is one. Hence, when $s \geqq f^{-1}(\sigma) \geqq \sigma$, the digits $\left(\nu_{s} / \tau\right)+1$ to $\nu_{s+1} / \tau$ of $x_{\tau}$ are the same as the digits 1 to $N_{s} / \tau$ of $U_{s}$. We shall now show that this is true.

The numbers $N_{s}$ referred to in $\S 3$ are chosen so that $\nu_{s} /(n \tau)$ is an integer, when $n \leqq f(s), \sigma \leqq f^{-1}(\sigma) \leqq s$ and $0<\rho \leqq 2^{2 \sigma-1}$, from which we see that $N_{s} /(n \tau)$ is an integer. Choosing $\alpha$ numbers from the set of equations (1) of $\$ 4$, where

* Since it is possible to choose $\alpha$ intervals from $\beta_{s}$ in $\beta_{g} C_{\alpha}$ ways, then for each choice it is possible to form $\beta_{8} C_{\alpha}$ numbers similar to the number which is given in the braces. The symbol

represents the symbolic sum $(v)$ of these numbers.

$$
\sum^{\beta_{s}} C_{\alpha}
$$

† Copeland, Admissible numbers in the theory of probability, American Journal of Mathematics, vol. 50, Oct., 1928, Theorem 16, p. 550.

† See von Mises, Vorlesungen aus dem Gebiete der angewandten Mathematik, pp. 147-48.

$\S$ See (3) and (4) of this section given below. 
$\rho \cdot 2^{s-\sigma}$ has been replaced by $\beta_{s}$, we may form the symbolic product $(\cdot)$ of these numbers and the negations of the remaining $\left(\beta_{s}-\alpha\right)$ numbers. This product $(\cdot)$ is

$$
\begin{gathered}
\prod_{i=1}^{\alpha}\left(\frac{q_{i}}{\beta_{s}}\right) X_{s} \cdot \prod_{i=\alpha+1}^{\beta_{8}} \sim\left(\frac{q_{i}}{\beta_{s}}\right) X_{8} \cdot=\cdot\left(\prod_{i=1}^{\alpha} X_{s}^{\left(q_{i}\right)} \cdot \prod_{i=\alpha+1}^{\beta_{8}}\left(1-X_{s}^{\left(q_{i}\right)}\right)\right), \\
\left(\prod_{i=1}^{\alpha} X_{s}^{\left(q_{i}+\beta_{8}\right)} \cdot \prod_{i=\alpha+1}^{\beta_{s}}\left(1-X_{s}^{\left(q_{i}+\beta_{s}\right)}\right)\right), \cdots
\end{gathered}
$$

We now raise the question when will

$$
\prod_{i=1}^{\alpha} X_{\theta}^{\left(q_{i}\right)} \cdot \prod_{i=\alpha+1}^{\beta_{\theta}}\left(1-X_{0}^{\left(q_{i}\right)}\right)
$$

be one? In order that this product be one, every $X_{i}{ }^{\left(q_{i}\right)}(i=1,2, \cdots, \alpha)$ must be one and every $X_{s}{ }^{\left(q_{i}\right)}\left(i=\alpha+1, \alpha+2, \cdots, \beta_{s}\right)$ must be zero. The only time this will be the case, is when there is a point in each of the $\alpha$ intervals, $q_{i} / \beta_{\text {s }}$ $(i=1,2, \cdots, \alpha)$, and not a point in the $\left(\beta_{s}-\alpha\right)$ intervals, $q_{i} / \beta_{s}(i=\alpha+1$, $\left.\alpha+2, \cdots, \beta_{s}\right)$, for the first period of the $s$-stage; that is, of the period $I_{\left(v_{\alpha} / \tau\right)+1}$. There are ${ }_{\beta} C_{\alpha}$ such numbers as (a) which can be formed from (1) of $\$ 4$, but if (a) has for its first digit one, the remaining $\left({ }_{\beta_{\alpha}} C_{\alpha}-1\right)$ numbers have for their digit zero and hence $U_{s}$ will have its first digit one. Hence the $\left[\left(\nu_{s} / \tau\right)+1\right]$ th digit of $x_{\tau}$ is the same as the first digit of $U_{s}$. This process may be continued for the $N_{s} / \tau$ digits of $U_{s}$.

We will now find the function $f(s)$ which has been referred to heretofore and the monotonic non-increasing sequence $\epsilon_{\mathrm{s}}$ approaching zero. We will show that $\eta(\rho, k, \sigma, \alpha, s)<\epsilon_{s} / 3$ for every $k, \rho, \alpha$ and $\sigma$ for which $k, \alpha, \sigma \leqq f(s)$ and $0<\rho \leqq 2^{2 \sigma-1}$, where

$$
\mid\left\{\beta _ { s } C _ { \alpha } \left(1-e^{\left.-\tau / \beta_{s}\right)^{\alpha}}\left(e^{\left.-\tau / \beta_{s}\right)^{\beta_{s}-\alpha}}\right\}^{k}-\left\{\left(\tau^{\alpha} e^{-\tau}\right) / \alpha !\right\}^{k} \mid=\eta(\rho, k, \sigma, \alpha, s) .\right.\right.
$$

If $k, \rho, \sigma$ and $\alpha$ are fixed, then the

$$
\lim _{s \rightarrow \infty} \eta(\rho, k, \sigma, \alpha, s)=0 .
$$

For every $\sigma$, there is a finite number of integers $\rho$, hence we may choose $\eta(k, \sigma, \alpha, s)$ greater than the greatest of the numbers $\eta(\rho, k, \sigma, \alpha, s)$. Then the $\eta(k, \sigma, \alpha, s)$ sequence dominates the $\eta(\rho, k, \sigma, \alpha, s)$ sequence, but it is not necessarily monotonic. Let $\epsilon(k, \sigma, \alpha, s)$ equal the least upper bound of the sequence $\eta(k, \sigma, \alpha, s), \eta(k, \sigma, \alpha, s+1), \cdots$, and now we have $\epsilon(k, \sigma, \alpha, s)$ dominating $\eta(k, \sigma, \alpha, s)$, which is a monotonic zero sequence. If for a given $\delta$, there exists a $z_{\delta}$ such that $z_{\delta+1}>z_{\delta}$ and $\epsilon\left(k, \sigma, \alpha, z_{\delta}\right) \leqq \epsilon(1,1,1, \delta)$, if $k \leqq \delta, \sigma \leqq \delta$, and $\alpha \leqq \delta$. Let $f(s)=\delta$ if $z_{\delta} \leqq s<z_{\delta+1}$. Hence $\epsilon(k, \sigma, \alpha, s) \leqq \epsilon(1,1,1, f(s))$ if $k$, 
$\sigma, \alpha \leqq f(s)$. Let $\epsilon_{s} / 3$ equal $\epsilon(1,1,1, f(s))$, from which it follows that $\eta(k, \sigma$, $\alpha, s)<\epsilon_{s} / 3$. Hence we conclude that

$$
\mid\left\{\beta_{s} C_{\alpha}\left(1-e^{\left.-\tau / \beta_{s}\right) \alpha}\left(e^{-\tau / \beta_{s}}\right)^{\beta_{s}-\alpha}\right\}^{k}-\left\{\left(\tau^{\alpha} e^{-\tau}\right) / \alpha !\right\} k \mid<\epsilon_{s} / 3,\right.
$$

if $k, \sigma, \alpha \leqq f(s)$ and $0<\rho \leqq 2^{2 \sigma-1}$.

We are now in a position to define the function $f^{-1}(\sigma)$. Let $f^{-1}(\delta)=z_{\delta}$. According to the definition of $z_{\delta}$, it follows that $f^{-1}(\delta+1)>f^{-1}(\delta)$ and since $f^{-1}(1) \geqq 1$, then $f^{-1}(\delta) \geqq \delta$. If $f^{-1}(\sigma) \leqq s<f^{-1}(\sigma+1)$, then $f(s)=\sigma$ and if $f^{-1}(\sigma)$ $\leqq s$, then $s \geqq f^{-1}(\sigma) \geqq \sigma$. Hence the function $f^{-1}(\sigma)$ has been established.

We shall now give the formal proof of Theorem 3; that is to say, we may now put it in form so that the fundamental lemma may be applied. From the fact that $U_{0}$ is an element of

we have

$$
A\left[{ }_{\beta_{\varepsilon}} C_{\alpha}\left(1-e^{-\tau / \beta_{s}}\right) \alpha\left(e^{-\tau / \beta_{s}}\right)^{\beta_{\varepsilon}-\alpha}\right],
$$

$$
p\left\{\prod_{i=1}^{k}\left(\frac{r_{i}}{n}\right) U_{\cdot} \cdot\right\}=\left\{{ }_{\beta_{s}} C_{\alpha}\left(1-e^{-\tau / \beta_{s}}\right)^{\alpha}\left(e^{\left.-\tau / \beta_{s}\right)_{s}-\alpha}\right\}^{k} .\right.
$$

It follows from ( $\left.1^{\prime}\right)$ and (2) that we can select the two sets of integers $M_{1}, M_{2}, \cdots, M_{s}, \cdots$ and $N_{1}, N_{2}, \cdots, N_{s}, \cdots$, referred to above, such that

$$
\left|p_{N}\left\{\prod_{i=1}^{k}\left(\frac{r_{i}}{n}\right) U_{s+1} \cdot\right\}-\left\{\frac{\tau^{\alpha} e^{-\tau}}{\alpha !}\right\}^{k}\right|<\frac{\epsilon_{s}}{3},
$$

if $N \geqq M_{\mathrm{o}} /(n \tau)$, where the sequence $M_{\mathrm{s}}$ is chosen so that $M_{\mathrm{s}} /(n \tau)$ is an integer, and

$$
\left|p_{N}\left\{\prod_{i=1}^{k}\left(\frac{r_{i}}{n}\right) U_{c} \cdot\right\}-\left\{\frac{\tau^{\alpha} e^{-\tau}}{\alpha !}\right\}^{k}\right|+\frac{\nu_{s}+M_{s}}{N_{s}}<\frac{\epsilon_{s}}{3},
$$

if $N \geqq N_{s} /(n \tau)$, where $\nu_{s}=N_{1}+N_{2}+\cdots+N_{s-1}$.

The inequalities (3) and (4) hold for every $k, n, \rho, \beta_{s}, f^{-1}(\sigma), \sigma, s, f(s)$ and $\alpha$ such that $k \leqq n \leqq f(s), 0<\rho \leqq 2^{2 \sigma-1}, \beta_{s}=\rho \cdot 2^{s-\sigma}, \sigma \leqq f^{-1}(\sigma) \leqq s, \sigma \leqq f(s), \alpha$ is less than or equal to the smaller of $\beta_{s}$ or $f(s)$, and for every set of numbers $r_{1}, r_{2}, \cdots, r_{k}$, such that $r_{i} \leqq n$ and $r_{i} \neq r_{j}$ if $i \neq j$.

When $\sigma \leqq f^{-1}(\sigma) \leqq s$, we have shown that the digits $\left(\nu_{s} / \tau\right)+1$ to $\nu_{s+1} / \tau$ of $x_{\tau}$ are the same as the digits 1 to $N_{s} / \tau$ of $U_{s}$. From the definition of $x_{\tau}$, we know that the digits 1 to $\nu_{\Gamma^{-1}(\sigma)} / \tau$ of $x_{\tau}$ are determined from the intervals $I_{1}$ to $I_{K}$ of the time axis, where $K=\nu_{f^{-1}(o)} / \tau$. The digits

$$
\frac{\nu_{s}}{n \tau}+1 \quad \text { to } \quad \frac{\nu_{s+1}}{n \tau} \quad \text { of } \quad\left[\prod_{i=1}^{k} \frac{r_{i}}{n} x_{\tau} \cdot\right]
$$


are the same as

$$
1 \text { to } \frac{N_{s}}{n \tau} \text { of }\left[\prod_{i=1}^{k} \frac{r_{i}}{n} U_{s} \cdot\right] .
$$

In applying the lemma, the numbers

$$
\begin{aligned}
& {\left[\prod_{i=1}^{k} \frac{r_{i}}{n} U_{s} \cdot\right],\left[\frac{\tau^{\alpha} e^{-\tau}}{\alpha !}\right]^{k}, \frac{M_{s}}{n \tau},} \\
& \frac{N_{s}}{n \tau}, \frac{\nu_{s}}{n \tau}, f^{-1}(\sigma) \text { and }\left[\prod_{i=1}^{k} \frac{r_{i}}{n} x_{\tau} \cdot\right]
\end{aligned}
$$

have taken the places of $U_{s}, p, H_{s}, J_{s}, \mu_{s}, s_{0}$ and $x$ respectively. Since the numbers $N$, have been selected, the time series is determined so that $x_{\tau}$ is a member of the set $A\left[\left(\tau^{\alpha} e^{-r}\right) / \alpha\right.$ ! ], for every $\rho, \sigma$ and $\alpha$.

8. We will now consider the period $\tau$ where sub-intervals of the form $2^{-\sigma+1}$ are omitted. With only slight modifications of the theorem in the preceding section, we can show that the laws of admissibility are satisfied for this case. The same type of time series will be used.

We shall consider $m$ intervals of the form $2^{-\sigma+1}$ which repeat every $\rho$ intervals. These intervals are of the same length as those intervals which make up that part of the time axis at the $\sigma$-stage. Let the intervals which are under consideration begin at $\rho_{i} \cdot 2^{-\sigma+1}(i=1,2, \cdots, m)$, where $\rho_{m}+1 \leqq \rho$ and $m \leqq \rho$. The sub-intervals of $I_{k}$ will be defined as $(k-1) \tau+\rho_{i} \cdot 2^{-\sigma+1}<h \leqq(k-1) \tau$ $+\left(\rho_{i}+1\right) 2^{-\sigma+1}(i=1,2, \cdots, m)$. We will define $x_{\tau^{\prime}}{ }^{*}$ such that its $k$ th digit is one if exactly $\alpha$ points lie in the $m$ intervals of $I_{k}$ and zero otherwise. Since the same type of time series is used here, then the series will be defined when the numbers $N_{s}$ are determined. Hence,

THEOREM 4. If the conditions $\left(H_{1}\right)$ of Theorem 1 hold, then the numbers $N_{1}, N_{2}, \cdots, N_{s}, \cdots$ can be chosen for every $\tau$ and $\tau^{\prime}$ satisfying the conditions

$$
\begin{aligned}
\tau & =\rho \cdot 2^{-\sigma+1} ; \\
\tau^{\prime} & =m \cdot 2^{-\sigma+1} ;
\end{aligned}
$$

$m, \rho$ and $\sigma$ are positive integers, where $m \leqq \rho$ and $0<\rho \leqq 2^{2 \sigma-1}$, so that the corresponding number $x_{\tau^{\prime}}$ is an element of $\left.A\left[\tau^{\prime \alpha} e^{-r^{\prime}}\right) / \alpha^{\prime}\right]$.

Let $\rho, \tau, \tau^{\prime}, \beta_{s}, m$ and $\alpha$ be defined such that $0<\rho \leqq 2^{2 \sigma-1}, \tau=\rho \cdot 2^{-\sigma+1}$, $\tau^{\prime}=m \cdot 2^{-\sigma+1}, \beta_{s}=m \cdot 2^{s-\sigma}, m \leqq \rho$ and $\alpha \leqq \beta_{8}$. At the $s$-stage there are $\beta_{8}$ intervals in the $m$ intervals of $I_{k}$. Let us consider an event which succeeds if there

* The symbol $x_{\tau^{\prime}}$ is used to denote the number $x\left(\alpha, \tau^{\prime}, t, \Lambda\right)$, where $\tau^{\prime}=m \cdot 2^{-\sigma+1}, t=0$ and $\Lambda=\tau=\rho \cdot 2^{-\sigma+1}$. 
is a point in each of $\alpha$ such intervals and no points in the remaining $\left(\beta_{\mathbf{s}}-\alpha\right)$ intervals. The number corresponding to this situation is

$$
W_{\bullet}=\sum^{\beta_{s} C_{\alpha}}\left\{\prod_{i k}^{\alpha}\left(\frac{q_{i k}}{\rho^{\circ-\sigma}}\right) X_{\varepsilon} \cdot \prod_{j n}^{\beta_{s}-\alpha} \sim\left(\frac{q_{j n}}{\rho \cdot 2^{s-\sigma}}\right) X_{\varepsilon} \cdot\right\} \vee,
$$

where $q_{i k}$ is of the form $2^{s-\sigma} \cdot \rho_{i}+k$ and $q_{j n}$ is of the form $2^{s-\sigma} \cdot \rho_{j}+n$, and if $i=j$, then $k \neq n$, and if $k=n$, then $i \neq j$. The numbers $i$ and $j$ take values from 1 to $m$ inclusive and the numbers $k$ and $n$ take values 1 to $2^{s-\sigma}$ inclusive.

This number $W_{\mathrm{o}}$ is admissible and is a member of the set

$$
A\left[{ }_{\beta_{s}} C_{\alpha}\left(1-e^{-\tau^{\prime} / \beta_{s}}\right) \alpha\left(e^{-\tau^{\prime} / \beta_{s}}\right)^{\beta_{s}-\alpha}\right] .
$$

When $s \geqq f^{-1}(\sigma) \geqq \sigma$ and $\nu_{s} / \tau<k \leqq \nu_{s+1} / \tau$, then there exist exactly $\alpha$ points in the $m$ intervals of $I_{k}$ if and only if the digit $\left(k-\nu_{s} / \tau\right)$ of $W_{s}$ is one. Hence, when $s \geqq f^{-1}(\sigma) \geqq \sigma$, the digits $\left(\nu_{s} / \tau\right)+1$ to $\nu_{s+1} / \tau$ of $x_{\tau^{\prime}}$ are the same as the digits 1 to $N_{s} / \tau$ of $W_{s}$. The numbers $N_{s}$ are chosen so that $\nu_{s} /(n \tau)$ is an integer, when $n \leqq f(s), \sigma \leqq f^{-1}(\sigma) \leqq s$ and $0<\rho \leqq 2^{2 \sigma-1}$, from which it follows that $N_{s} /(n \tau)$ is an integer.

As in Theorem 3, we can find a function $f(s)$ and a monotonic non-increasing sequence $\epsilon_{B}$ whose limit is zero, such that

$$
\mid\left\{{ }_{\beta_{s}} C_{\alpha}\left(1-e^{\left.-\tau^{\prime} / \beta_{s}\right) \alpha}\left(e^{-\tau^{\prime} / \beta_{s}}\right)^{\beta_{s}-\alpha}\right\}^{k}-\left\{\left(\tau^{\prime \alpha} e^{-\tau^{\prime}}\right) / \alpha !\right\}^{k} \mid<\epsilon_{s} / 3,\right.
$$

where $k, \alpha, \sigma \leqq f(s), m \leqq \rho$ and $0<\rho \leqq 2^{2 \sigma-1}$.

Since $W$, is a member of the set

$$
A\left[{ }_{\beta_{\varepsilon}} C_{\alpha}\left(1-e^{-\tau^{\prime} / \beta_{s}}\right)^{\alpha}\left(e^{-\tau^{\prime} / \beta_{s}}\right)^{\beta_{s}-\alpha}\right],
$$

we know that

$$
p\left\{\prod_{i=1}^{k}\left(\frac{r_{i}}{n}\right) W_{\bullet} \cdot\right\}=\left\{{ } _ { \beta _ { s } } C _ { \alpha } \left(1-e^{\left.-\tau^{\prime} / \beta_{s}\right) \alpha}\left(e^{\left.-\tau^{\prime} / \beta_{s}\right)_{\beta_{t}-\alpha}}\right\}^{k} .\right.\right.
$$

It follows from (a) and (b) that we can select two sets of integers $M_{1}$, $M_{2}, \cdots, M_{s}, \cdots$ and $N_{1}, N_{2}, \cdots, N_{s}, \cdots$ such that

$$
\left|p_{N}\left\{\prod_{i=1}^{k}\left(\frac{r_{i}}{n}\right) W_{\bullet+1} \cdot\right\}-\left\{\frac{\tau^{\prime \alpha} e^{-\tau \prime}}{\alpha !}\right\}^{k}\right|<\frac{\epsilon_{s}}{3},
$$

when $N \geqq M_{8} /(n \tau)$, where the sequence $M_{8}$ has been chosen so that $M_{8} /(n \tau)$ is an integer, and

$$
\left|p_{N}\left\{\prod_{i=1}^{k}\left(\frac{r_{i}}{n}\right) W_{0} \cdot\right\}-\left\{\frac{\tau^{\prime \alpha} e^{-\tau^{\prime}}}{\alpha !}\right\}\right|+\frac{\nu_{s}+M_{s}}{N_{s}}<\frac{\epsilon_{s}}{3},
$$

if $N \geqq N_{s} /(n \tau)$, where $\nu_{s}=N_{1}+N_{2}+\cdots+N_{s-1}$. 
It is understood that conditions (c) and (d) hold for every $\rho_{1}, \rho_{2}, \cdots, \rho_{m}$, $\rho, n, m, k, \sigma, s, f^{-1}(\sigma), \beta_{s}$ and $\alpha$ such that $k \leqq n \leqq f(s), 0<\rho \leqq 2^{2 \sigma-1}, m \leqq \rho$, $\sigma \leqq f(s), \rho_{m}+1 \leqq \rho, \sigma \leqq f^{-1}(\sigma) \leqq s, \beta_{s}=m \cdot 2^{s-\sigma}, \alpha$ is less than or equal to the smaller of $\beta_{s}$ or $f(s)$, and for every set of numbers, $r_{1}, r_{2}, \cdots, r_{k}$, such that $r_{i} \leqq n$ and $r_{i} \neq r_{j}$ if $i=j$.

It follows from the definition of $x_{\tau^{\prime}}$ that the digits $\nu_{s} /(n \tau)+1$ to $\nu_{s+1} /(n \tau)$ of $\left[\Pi_{i=1}^{k}\left(r_{i} / n\right) x_{\tau^{\prime}} \cdot\right]$ are the same digits as 1 to $N_{s} /(n \tau)$ of $\left[\Pi_{i=1}^{k}\left(r_{i} / n\right) W_{s} \cdot\right]$.

Hence, in applying the lemma to this theorem, we see that the numbers $N_{s}$ have been selected so that $x_{\tau^{\prime}}$ is an element of the set $A\left[\left(\tau^{\prime \alpha} e^{-r^{\prime}}\right) / \alpha !\right]$, for every $m, \rho, \sigma$ and $\alpha$, where $m \leqq \rho$.

If $m=\rho$, then $\rho_{1}=0$ and this theorem becomes Theorem 3. Furthermore, if $\rho_{1}=r$ and $\rho_{m}=r+m-1$, the number $x_{\tau^{\prime}}$ becomes $x(\alpha, \tau, t, \Lambda)$, where $t=r \cdot 2^{-\sigma+1}, \tau=m \cdot 2^{-\sigma+1}$ and $\Lambda=\rho \cdot 2^{-\sigma+1}$.

9. Since the sequence of numbers $N_{8}$ has been found, we have constructed a time series which is consistent with the frequency theory of probability.

It may be noted that several theorems proved in Professor A. H. Copeland's work (American Journal of Mathematics, vols. 50, 51, and 53), can be proved by applying the fundamental lemma in this paper.

ST. LOUIS UNIVERSITY,

ST. LouIs, Mo. 